\title{
Effects of physical activity on sustained attention: a systematic review
}

\section{Efeitos da atividade física na atenção sustentada: uma revisão sistemática}

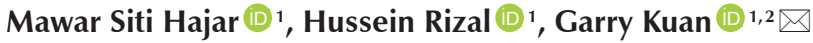 \\ ${ }^{1}$ Universiti Sains Malaysia, Exercise and Sports Science, School of Health Sciences. Kubang Kerian, Kelantan, Malaysia. \\ ${ }^{2}$ Brunel University London, Department of Life Sciences, United Kingdom.
}

How to cite this article:

Hajar MS, Rizal H, Kuan G. Effects of physical activity on sustained attention: a systematic review. Sci Med. 2019;29(2):e32864. http://doi. org/10.15448/1980-6108.2019.2.32864

\section{ABSTRACT}

AIM: Physical activity is known for its benefits to improve cognitive functions such as sustained attention. The study aimed to conduct a systematic review on the effects of physical activity on sustained attention.

METHODS: Literature search was conducted using databases from Scopus, PubMed, ResearchGate, ScienceDirect and Google Scholar. The date of these published articles ranged from January 2010 to August 2018.

RESULTS: A total of thirteen studies using the longitudinal design was selected to be part of this review. Most studies showed positive relationships between physical activity and sustained attention.

CONCLUSIONS: Future studies may consider a longitudinal study design to study the effect of physical activity on sustained attention in prolonged time; as well as the corresponding affect with gender, age, motivation and to investigate the relationship between physical activities together with other possible factors that can indirectly affect sustained attention.

KEYWORDS: physical activity, exercise, sustained attention, vigilance

\section{RESUMO}

OBJETIVO: A atividade física é conhecida por seus benefícios para melhorar as funções cognitivas, como a atenção sustentada. O estudo objetivou realizar uma revisão sistemática sobre os efeitos da atividade física na atenção sustentada.

MÉTODOS: A pesquisa bibliográfica foi realizada utilizando os bancos de dados da Scopus, PubMed, ResearchGate, ScienceDirect e Google Scholar, com artigos publicados entre janeiro de 2010 e agosto de 2018.

RESULTADOS: Treze estudos com delineamento longitudinal foram selecionados para fazer parte desta revisão. A maioria dos estudos mostrou relações positivas entre atividade física e a atenção sustentada.

CONCLUSÕES: Futuros estudos poderão considerar um delineamento longitudinal para investigar o efeito da atividade física sobre a atenção sustentada por tempo prolongado, assim como sua relação pode ser afetada por sexo, idade e motivações, e, relacionar as atividades físicas com outros possíveis fatores que podem afetar indiretamente a atenção sustentada.

DESCRITORES: atividade física; exercício; atenção sustentada; vigilância. 
Abbreviations: PA, Physical activity; PVT, Psychomotor Vigilance Test.

\section{INTRODUCTION}

Physical activity (PA) is any bodily movement produced by the skeletal muscles resulting in energy consumption [1] or any bodily movement produced by the muscular system that increases energy expenditure above the normal physiological demands [2]. Whereas, exercise is defined as a PA that is planned, structured, and aims to improve or maintain one or more components of physical fitness [1] and is considered as a subcategory of PA. According to the World Health Organization in 2010, children and adolescents aged between 5-17 years old are recommended to accumulate at least 60 minutes of moderate-to-vigorous physical activity daily [3]. Meanwhile, guidelines by American College of Sports Medicine and American Heart Association in 2007, adults aged between 18-65 years old are recommended to participate in moderateintensity endurance PA, for a minimum 30 minutes, five days per week; or participate in vigorous-intensity endurance PA for a minimum 20 minutes, three days per week to maintain a healthy lifestyle.

It is well known that PA can benefit us physically by increasing physical fitness and promote the development of both the cardiovascular and musculoskeletal systems [4]. However, PA is also known to improve us mentally by enhancing learning and memory [5], improve academic achievement [6] and even enhance an individual's quality of life-based on previous systematic reviews and meta-analysis $[7,8]$. PA can improve a persons' cognitive functioning. Recently, there are some reviews conducted to examine the relationship between PA and cognitive functions such as working memory [9], and attention [10]. However, fewer reviews have been conducted to examine the relationship between PA and sustained attention. It may be due to the lack of updated knowledge and interest in research that has been conducted on this topic. Since then, this topic has become an area of interest to us to find out the extent to which exercise gives cognitive benefits to sustained attention.

Sustained attention is a process that enables sustained performance on tasks over an extended period [11], while Sarter et al. [12] defined it as a fundamental component of attention characterised by the readiness to detect rarely and unpredictably signals over prolonged periods of time. Both authors have highlighted the significant characteristic of sustained attention that is to sustain activity or performance over a prolonged time. It is one of the primary elements of the attention process that enables a person to maintain vigilance, continuous effort, persistence response, selective, and focused attention.

In assessing sustained attention ability, there are many types and versions of sustained attention tests. The most frequent test used was the Psychomotor Vigilance Test (PVT) [13-18], followed by the Continuous Performance Test and its many versions such as Identical Pairs [19], and Conners'-II [20]. Other sustained attention tests are Digit Vigilance Test [21]; Picture Deletion Task Performance [22]; Rapid Visual Processing [23]; and Visual Pursuit Test [24].

A previous study conducted by Luque-Casado et al. [25], stated that those who have higher physical fitness were related to better overall sustained attention, demonstrating a better ability to allocate attentional resources over time. Besides, activities involving prolonged high demand attention such as military, surgery, lifeguarding; and is also needed in many daily activities such as attending academic lessons or examinations at school. Moreover, this cognitive function represents a fundamental component of the general cognitive capacities of the human because a reduced ability to monitor significant sources of the information, which will affect all cognitive abilities such as slow or failures to respond to target stimuli [12].

However, in nature, a human cannot maintain their optimal attention for a long time without falling into a fatigued state [26]. Mental fatigue may affect the attention that can decrease the ability to suppress irrelevant information and inhibit shifts of attention to irrelevant stimuli, which leads to the increase in reaction time and the number of incorrect responses $[27,28]$. Those that have difficulty in sustaining their attention may appear to be daydreamy, bored, fidgety, and disorganised. In the worst case, it may result in attentiondeficit hyperactivity disorder. There are several ways to overcome mental fatigue such as drinking enough water [29]; listening to smooth music [30]; getting enough sleep, as well as being physically active [19]. PA not only helps to overcome a state of mental fatigue but also improves sustained attention especially in long duration activity such as during school hours [31,32]. It has been addressed in many research such as Palmer et al. [22] found that acute exercise enhanced sustained attention in pre-schoolers as indexed by Picture Deletion Task Performance omission and it aligns with the extant literature regarding older children, 
aged 13 to 16 years old [17] and second, third and fourth grade [33]. In sport performance perspective, a study by Ballester et al. [13] found that vigilance capacities by athletes were much better than nonathletes. They responded faster with fewer lapses and able to maintain a similar level of performance throughout PVT task compared to non-athletes. Interestingly the between-group difference vigilance capacities did not seem to be influenced by motivation toward the task and/or perceived activation prior to the task, though the two groups significantly differed in these two measures. Medina et al. [20] found that there is increased surveillance, decreased impulsivity, increased reaction speed, and greater stability in all the metrics of sustained attention after exercise and stretching among attention-deficit hyperactivity disorder subjects, ignoring methylphenidate use. The methylphenidate is known as a drug that has been used for attention-deficit hyperactivity disorder treatment. However, in other research [34] they found contrary finding that aerobic exercise had no effects on sustained attention among female students with a mean age of 16 years old.

Based on the diversity of findings above, it shows the need for a comprehensive and systematic review to obtain a transparent and independent decision against prejudice risk. Therefore, some questions need to be answered among which are what is the minimum or the maximum number of participants recruited and at what age? What interventions are given to participants to examine the impact of physical activity on their sustained attention? How about the sustained attention comparison between physically active individuals and physically inactive individuals, is there any significant difference? What is the result of the study or intervention given? What is the design of the study which most researchers implemented?

Therefore, this paper attempts to write a systematic review to answer related questions on the effects of PA on sustained attention and whether other interventions in addition to PA may have improved effects on sustained attention. As authors, we hope this paper will help researchers who are interested in studying this field.

\section{METHODS}

Based on PRISMA guidelines, eligibility criteria are divided into two; study characteristics and report characteristics. This is important so that the review produced are authentic and valid as well as easier for readers to keep up with the issue. Eligible study characteristics were: (1) interventional studies to retrieve stronger data in the form of experimental based designs for this systematic review; (2) conducted among healthy children, adults or special population regardless of races; (3) those reported an association between PA and sustained attention or effects of PA in combination with other interventions on sustained attention. Meanwhile, eligible report characteristics were: (1) those published in English; (2) publication range from January 2010 till August 2018 (ranged from the past eight years of released publication) as there were more new and updated knowledge regarding PA on sustained attention are published; (3) only published articles (on dates mentioned above). Review paper, meta-analysis, validation studies, dissertations, thesis, conference abstracts, monographs, commentaries, and brief reports were not included to narrow down the search category.

We developed a data extraction sheet (based on the Cochrane Consumers and Communication Review Group's data extraction template), pilot-tested it on all included studies, and refined it accordingly. One review author extracted the following data from included studies while a second review author checked the data. There was a discussion among two review authors to resolve disagreement. However, if no agreement could be reached, third author would decide. Several information or data that we extract from selected studies were: a) characteristics of participants (number, type of population, gender and age); b) PA intervention or test, as well as its frequency and number of sessions performed; c) sustained attention assessment and; d) results of the study. A two-phase strategy was applied to search for potential studies. Firstly, in literature search, the databases used were Scopus, PubMed, ResearchGate, ScienceDirect and Google Scholar. Search strategies included the combination "AND" of variations between two groups of keywords including (1) PA; exercise, (2) sustained attention; vigilance. The second phase, titles and abstracts of potential studies were screened by the researchers. References cited by each of journals were examined to identify other potential studies for inclusion.

The Figure 1 shows a forest plot of comparison between low levels versus moderate-to-high level physical activity. The average effect size of all studies is $d=1.29$. According to a common interpretation of effect sizes, this would suggest that the intervention being tested in all studies had a large effect size. In other words, it worked and had a large effect. However, the heterogeneity of study is $\mathrm{I}^{2}=42 \%$ in which $42 \%$ less confident on the intervention to consistently have a large effect. 
Figure 2 shows the risk of bias collected from all 13 studies presented in the form of a graph. The studies in this review are predominantly quasi-experimental, therefore the random sequence generation has a higher risk for bias. In addition, larger study designs conducted outside of clinical labs would also generally be harder to blind and conceal. Hence, these scales show a greater percentage of high risk of bias. The blinding of outcome assessment is also intrusive which is evident in Figure 2 as researchers are required to be present during the handling and administration of instruments, mainly questionnaires, and tests. Incomplete outcome data shows almost equal percentages in a lower risk of bias and unclear risk of bias as participants in these studies generally are accounted for until the end of the study. For example, the studies by Luque-Casado et al. $[17,25]$, withdrawal of participants were clearly stated with data reduction section stating cut-off point as the main reason. Finally, the results for both significant and non-significant results were clearly reported in the results section for all the included studies.

\section{RESULTS}

The Figure 3 shows the inclusion diagram for the effect of PA and sustained attention based on the

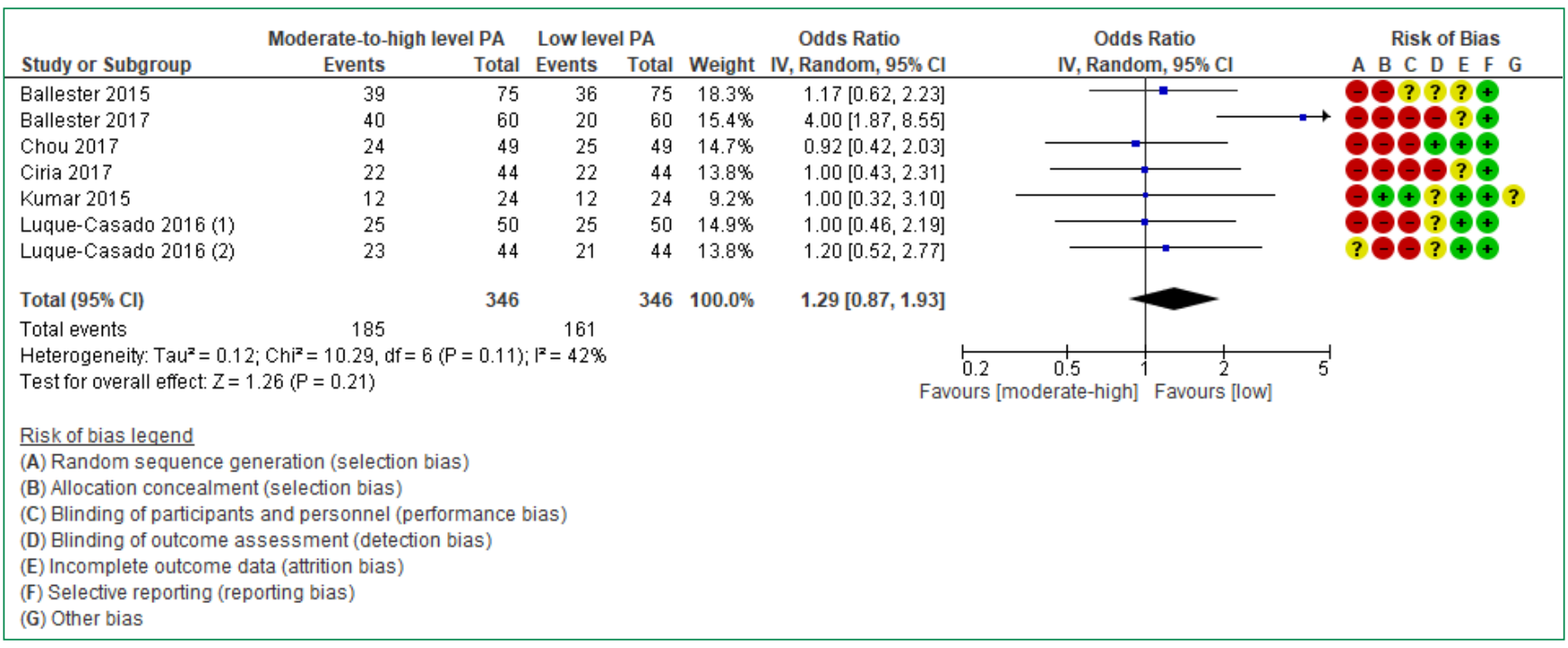

Figure 1. Forest plot of comparison: Low level PA versus Moderate-to-high level PA.

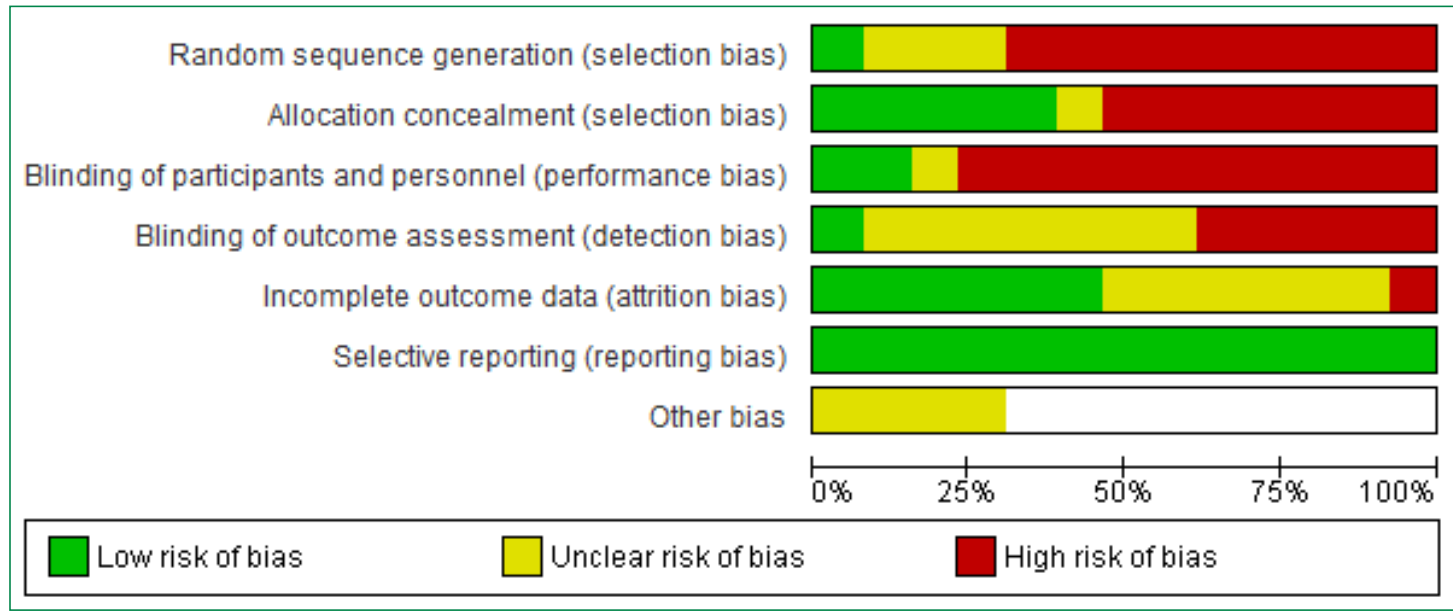

Figure 2. Risk of bias graph: review authors' judgements about each risk of bias item presented as percentages across all included studies. 
PRISMA guideline. A total of 1254 search results were found in which only 34 studies were found to be relevant to the research question posed in this paper, which is to: determine the effects of PA and other combining interventions on sustained attention. Out of the 34 studies, 19 studies were removed during the abstract/method as it did not measure the effects of PA and sustained attention directly. Afterward, a full copy of paper was obtained for those that met initial screening criteria and then fully examined by the researchers using triangulation method; which facilitates validation of data through cross verification from two or more sources or research technique.

After full reading, two additional papers by Roland [32] and Kalia [34] were excluded due to having no reference and being a thesis dissertation. The following data were extracted from all eligible articles: (a) year of publication and authors, (b) study design, (c) sample size, (d) age, (e) PA intervention/ assessment, (f) sustained attention assessment, and (g) main outcomes. The Table 1 summarises the authors and year of study, study design, participants' characteristics recruited, PA and sustained attention assessments, control variables, and the results. All information is obtained after a full reading and most

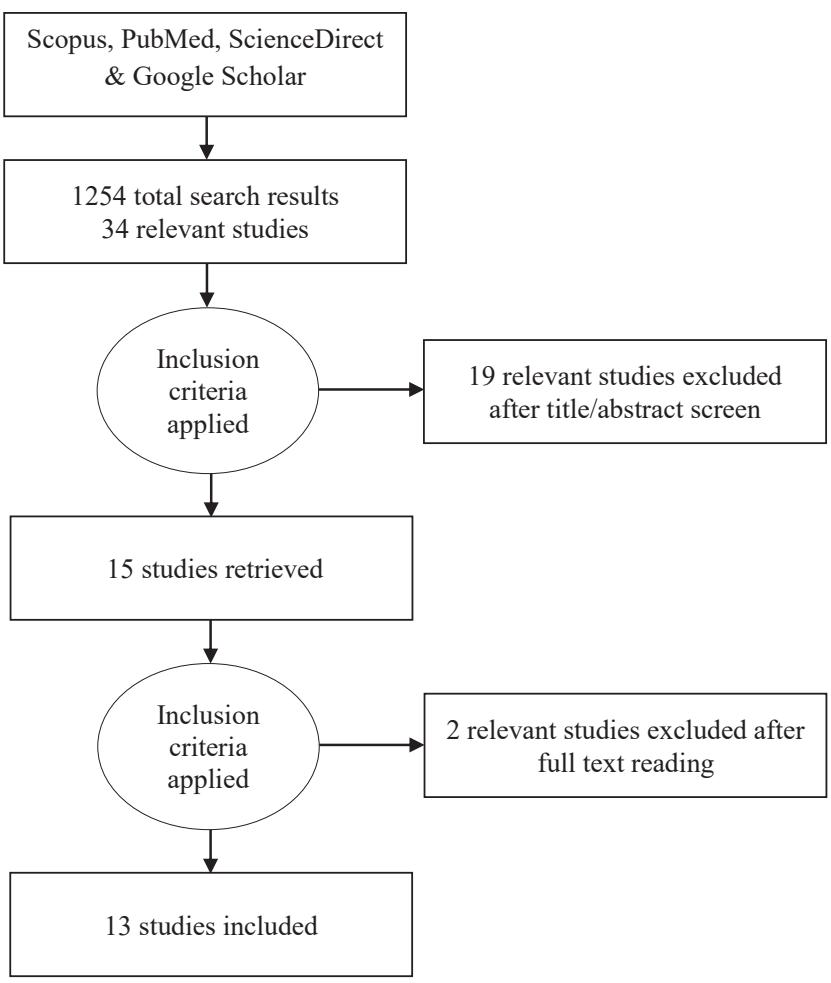

Figure 3. Inclusion diagram for effect of physical activity on sustained attention. of it is extracted from the method section as well as the results of the study. A total of thirteen studies were included in the final review.

The total number of studies that met the inclusion criteria and were subsequently included is thirteen. Most of the studies examined the effect of PA on sustained attention among the younger population. To achieve a significant result, the young population is easier to be recruited in large samples compared to the older population. Besides, the smaller sample size may also reduce the power of the study as well as increases the margin of error, which can lead to less significant result [35]. Young population can be recruited either from school or higher education institutes.

Here we explain answers to each of the research questions given above to gain more insights about included studies: 1 ) what is the minimum or maximum number of participants recruited and at what age? In Figure 4, the largest sample size recruited was 423 participants which were from a primary school. The trend in sample size decreases following the incremental in the participant's age. The studies were divided into three groups by the age of participants recruited, namely; children (4-12 years old), adolescent (13-16 years old), and young adults (22-27 years old). 2) What interventions are given to participants to examine the impact of physical activity on their sustained attention? In Figure $\mathbf{4}$ also shown specific intervention of physical activity given to participants. Specifically, most studies provide moderate to high-intensity aerobic exercise interventions for 30 to 60 minutes per session and were done two to three times a week (Figure 5). 3) How about the sustained attention comparison between physically active individuals and physically inactive individuals, is there any significant difference? In general, most of physically active individuals have shown better result in sustained attention compared to less physically active or sedentary individuals. In fact, they showed shorter reaction time, increase surveillance and less impulsive as well as improve in overall cognitive functioning. 4) What is the result of the study or intervention given? Most of studies have shown the positive relationship between physical activity and sustained attention. 5) What is the design of the study which most researchers implemented? Most of studies implemented quasi-randomised controlled trials (either pretest-posttest design, nonequivalent design, interrupted time series design or combination design), one study used cross-sectional design [23], one study used non-randomised controlled study design [24], and only one study implemented randomised controlled trials [19]. 
Table 1. Intervention characteristics and result of studies.

\begin{tabular}{|c|c|c|c|c|}
\hline Authors (year) & $\begin{array}{l}\text { Participants characteristic: } \\
\text { N, Mean (SD) \& age }\end{array}$ & $\begin{array}{l}\text { Physical activity } \\
\text { intervention/test, } \\
\text { frequency \& total sessions }\end{array}$ & $\begin{array}{l}\text { Sustained attention } \\
\text { assessment }\end{array}$ & Results \\
\hline $\begin{array}{l}\text { Medina et al. } \\
(2010)[20]\end{array}$ & $\begin{array}{l}\mathrm{N}=25 \text { males } \\
\text { (MTP user (US), } \mathrm{n}=16 \& \\
\text { non-users (NUS), } \mathrm{n}=9) \\
\text { Age: } \\
\text { US }=9.33(2.87) \text { years old } \\
\text { NUS }=9.75(2.38) \text { years old }\end{array}$ & $\begin{array}{l}\text { Treadmill running } \\
\text { (moderate-to vigorous } \\
\text { intensity) } \\
\text { Frequency: once/session } \\
\text { Total sessions: } 1\end{array}$ & $\begin{array}{l}\text { Corner's Continuous } \\
\text { Performance Test-II } \\
\text { (CPT-II) }\end{array}$ & 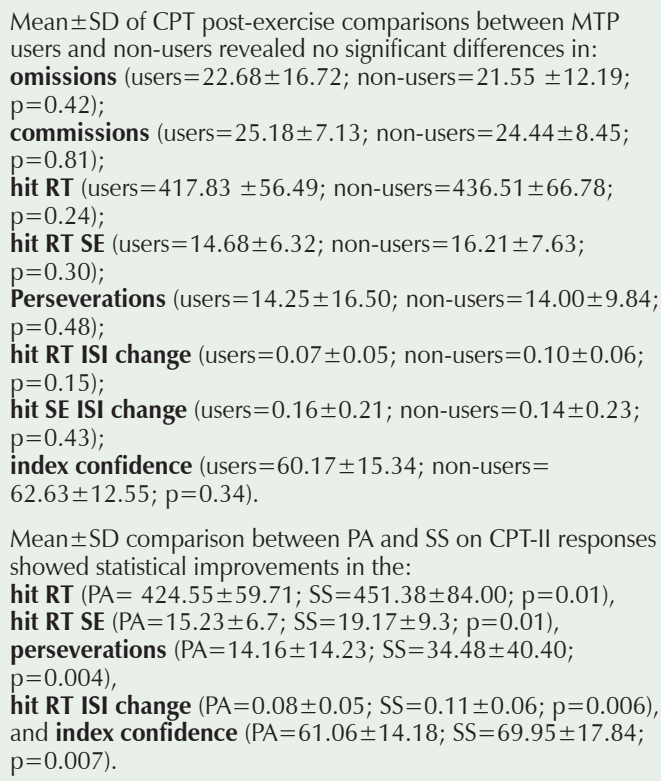 \\
\hline $\begin{array}{l}\text { Palmer et al. } \\
(2013)[22]\end{array}$ & $\begin{array}{l}\mathrm{N}=16 \text { (males, } n=13 \text { \& } \\
\text { females, } n=3) \\
\text { Age: } 49.4 \text { (5.3) months }\end{array}$ & $\begin{array}{l}30 \text { minutes movement } \\
\text { program (accelerometer) } \\
\text { Frequency: once/day } \\
\text { Total sessions: } 3 \text { ( } 3 \text { days) }\end{array}$ & $\begin{array}{l}\text { Picture Deletion Task } \\
\text { for Pre-schoolers } \\
\text { (PDTP) }\end{array}$ & $\begin{array}{l}\text { Omission: Wilcoxon signed-rank test revealed a significant } \\
\text { effect for condition }(p=0.01) \text { with fewer omission was made } \\
\text { after the exercise condition, mean }(S D)=25.6(12.3) \text { than after } \\
\text { sedentary condition, mean }(S D)=44.3(28.7) \text {. } \\
\text { Commission: Wilcoxon signed-rank test revealed no significant } \\
\text { effect for condition ( } p=0.06) \text { though fewer commission was } \\
\text { made after exercise condition, mean }(S D)=4.88(4.43) \text { than } \\
\text { after the sedentary condition, mean }(S D)=10.6(17.2) \text {. }\end{array}$ \\
\hline $\begin{array}{l}\text { Ballester et al. } \\
(2015)[13]\end{array}$ & $\begin{array}{l}\mathrm{N}=75 \text { (athletes, } \mathrm{n}=39 \text { \& } \\
\text { non-athletes, } \mathrm{n}=36) \\
\text { Age: } \\
\text { Athletes }=13.7(0.5) \\
\text { years old } \\
\text { Non-athletes=13.8 }(0.6) \\
\text { years old }\end{array}$ & $\begin{array}{l}\text { Leger Multi-stage fitness } \\
\text { test } \\
\text { Frequency: once/session } \\
\text { Total sessions: } 1\end{array}$ & $\begin{array}{l}\text { Psychomotor } \\
\text { Vigilance test (PVT) }\end{array}$ & $\begin{array}{l}\text { Mean RTs revealed significant main effects of sport } \\
\text { participation, } F(1,71)=17.27, p<0.001, \eta^{2} \text { partial }=0.19 \text { and } \\
\text { time on task, } F(1.50,106.67)=5.84, p=0.03, \eta^{2} \text { partial }=0.08 \text {. } \\
\text { Participants' lapses revealed significant main effects of time on } \\
\text { task, } F(1.71,122)=7.41, p<0.001, \eta^{2} \text { partial }=0.09 \text {, and sport } \\
\text { participation, } F(1,71)=15.08, p<0.001, \eta^{2} \text { partial }=0.04 \text {. }\end{array}$ \\
\hline $\begin{array}{l}\text { Leong et al. } \\
(2015) \text { [21] }\end{array}$ & $\begin{array}{l}\mathrm{N}=81 \text { (only females) } \\
\text { Age: (16 years old) }\end{array}$ & $\begin{array}{l}\text { One-hour aerobic dance } \\
\text { Frequency: twice/week } \\
\text { Total sessions: } 12 \text { ( } 6 \text { weeks) }\end{array}$ & Digit Vigilance Test & $\begin{array}{l}\text { No significant interaction between experimental groups across } \\
\text { testing sessions on sustained attention total time and errors } \\
\text { for both digits } 6(p=0.81, p=0.23) \text {; and digits } 9(p=0.45 \text {, } \\
p=0.06) \text {. }\end{array}$ \\
\hline $\begin{array}{l}\text { Kumar et al. } \\
(2015)[55]\end{array}$ & $\begin{array}{l}\mathrm{N}=24 \text { ( } 16 \text { males and } 8 \\
\text { females) } \\
\text { Age: } \\
\text { Endurance-trained: } 27.7 \\
\text { (5.5) years old } \\
\text { Sedentary: } 26.8(7.0) \\
\text { years old }\end{array}$ & $\begin{array}{l}30 \text { minutes moderate } \\
\text { cycling and/or to volitional } \\
\text { fatigue } \\
\text { Frequency: once/session } \\
\text { Total sessions: } 7 \text { including } \\
2 \text { preliminary and } \\
\text { familiarisation sessions }\end{array}$ & $\begin{array}{l}\text { Continuous } \\
\text { Performance Task } \\
\text { (CPT) }\end{array}$ & $\begin{array}{l}\text { Accuracy: There was a significant condition } x \text { time interaction } \\
\text { in both groups ( } 30 \text { minutes moderate exercise vs rest control), } \\
F(2,2)=4.23, p=0.02, \eta^{2}=0.19 \text {. } \\
\text { However, it declined }(p<0.001) \text { from baseline CPT to post CPT } \\
\text { prior to } 30 \text { minutes rest (relative } \triangle \text { score of }-4.5 \pm 4.6 \% \text { ) and } \\
\text { prior to exercise }(-5.0 \pm 4.7 \%) \text {. } \\
\text { Compared to post CPT, improvement in accuracy was higher } \\
\text { ( } p=0.03) \text { after exercise as compared to after rest. } \\
\text { Precision: There was a significant main effect for time, } \\
F(2,36)=12.04 \text {, } p<0.001, \eta^{2}=0.40 \text { and a significant condition } \\
x \text { time interaction, } F(2,23)=5.25, p=0.02, \eta^{2}=0.24 \text {. } \\
\text { However, it declined significantly ( } p<0.001) \text { during initial } 20 \\
\text { minutes CPT in the trial preceding the exercise condition but } \\
\text { not prior to rest }(p=0.08) \text {. } \\
\text { Compared to post CPT, improvement in precision was higher } \\
(p=0.03) \text { after exercise compared to rest. }\end{array}$ \\
\hline $\begin{array}{l}\text { Luque-Casado } \\
\text { et al. (2016) [16] }\end{array}$ & $\begin{array}{l}\mathrm{N}=42 \text { (higher fit, } \mathrm{n}=22 \text { \& } \\
\text { lower fit, } \mathrm{n}=20) \\
\text { Age: } \\
\text { mean }(95 \% \text { of } \mathrm{Cl}) \text { : } \\
\text { higher fit }=22(21,24) \\
\text { years old } \\
\text { lower fit }=23(22,24) \\
\text { years old }\end{array}$ & $\begin{array}{l}\text { Incremental effort test } \\
\text { Frequency: once/session } \\
\text { Total sessions: } 1\end{array}$ & $\begin{array}{l}\text { Psychomotor } \\
\text { Vigilance Test (PVT) }\end{array}$ & $\begin{array}{l}\text { The cue-locked ERP pairwise comparisons (FDR corrected; } \\
p \text { threshold=0.029) showed significant differences between } \\
\text { high-fit group and lower-fit group at block } 1(p<0.01) \text {, block } 2 \\
(p<0.01) \text { and block } 3(p=0.029) \text {. } \\
\text { Target-locked ERP analysis revealed that the comparisons } \\
\text { between blocks in the lower-fit group showed significant } \\
\text { difference only between block } 1 \text { and } 5(p=0.003) \text {. }\end{array}$ \\
\hline
\end{tabular}


Table 1 (conclusion)

\begin{tabular}{|c|c|c|c|c|}
\hline Authors (year) & $\begin{array}{l}\text { Participants characteristic: } \\
\text { N, Mean (SD) \& age }\end{array}$ & $\begin{array}{l}\text { Physical activity } \\
\text { intervention/test, } \\
\text { frequency \& total sessions }\end{array}$ & $\begin{array}{l}\text { Sustained attention } \\
\text { assessment }\end{array}$ & Results \\
\hline $\begin{array}{l}\text { Luque-Casado } \\
\text { et al. (2016) [17] }\end{array}$ & $\begin{array}{l}\mathrm{N}=44 \text { (high-fit, } \mathrm{n}=23 \text { \& } \\
\text { low-fit, } \mathrm{n}=21) \\
\text { Age: } \\
\text { mean }(95 \% \text { of } \mathrm{Cl}) \text { : } \\
\text { High-fit: } 23(21,24) \\
\text { years old } \\
\text { Low-fit: } 23(22,24) \\
\text { years old }\end{array}$ & $\begin{array}{l}\text { Submaximal } \\
\text { cardiorespiratory fitness } \\
\text { test } \\
\text { Frequency: once/session } \\
\text { Total sessions: } 1\end{array}$ & $\begin{array}{l}\text { Psychomotor } \\
\text { Vigilance Task (PVT) }\end{array}$ & $\begin{array}{l}\text { Mean reaction time: } \\
\text { There were significant main effects of group ( } p=0.001 \text { ) and } \\
\text { time-on-task }(p=0.001) \text {. } \\
\text { The interaction between both factors also reached statistical } \\
\text { significant }(p=0.001) \text {. } \\
\text { Pairwise comparisons between groups were performed within } \\
\text { each temporal block (FDR corrected; } p \text {-threshold }=0.009 \text { ). } \\
\text { The comparisons showed significant differences between } \\
\text { groups at block } 1 \text { and } 2 \text { (both } p s=0.001 \text { ) with high-fit being } \\
\text { faster than low-fit group. } \\
\text { However, there were no significant differences when } \\
\text { comparing groups at blocks } 3 \text { and } 4 \text { (both } p s=0.18 \text { ), then } \\
\text { between groups differences reached again statistical significance } \\
(p=0.009 \text { ) at block } 5 \text {, but with the high-fit being slower than } \\
\text { low-fit group. }\end{array}$ \\
\hline $\begin{array}{l}\text { Wilson et al. } \\
\text { (2016) [18] }\end{array}$ & $\begin{array}{l}\mathrm{N}=58 \text { (only males) } \\
\text { Age: }(11-12 \text { years old) }\end{array}$ & $\begin{array}{l}\text { Active Lesson Breaks } \\
\text { (ALBs) and Passive Lesson } \\
\text { Breaks (PLBs) measured } \\
\text { with accelerometer } \\
\text { Frequency: once/day } \\
\text { ( } 3 \text { out of } 5 \text { school days) } \\
\text { Total sessions: } 12 \text { (4 weeks) }\end{array}$ & $\begin{array}{l}\text { Psychomotor } \\
\text { Vigilance Test (PVT) }\end{array}$ & $\begin{array}{l}\text { Mean RTs and \% lapses revealed the ALBs and PLBs condition } \\
\text { was not significant }(p=0.61, p=0.64) \text {. }\end{array}$ \\
\hline $\begin{array}{l}\text { Geertsen et al. } \\
\text { (2016) [23] }\end{array}$ & $\begin{array}{l}\mathrm{N}=423 \text { (males and females) } \\
\text { Age: (8-10 years old) }\end{array}$ & $\begin{array}{l}\text { Visuomotor accuracy- } \\
\text { tracking task } \\
\text { Frequency: } 10 \text { trials/ } \\
\text { session (including } 3 \\
\text { practice trials) } \\
\text { Whole-body coordination } \\
\text { task } \\
\text { Frequency: once/session } \\
\text { Yo-yo intermittent } \\
\text { recovery level } 1 \text { children's } \\
\text { test (YYIR1C) } \\
\text { Frequency: once/session } \\
\text { Total sessions: } 3\end{array}$ & $\begin{array}{l}\text { Rapid Visual } \\
\text { Processing (RVP) } \\
\text { from Cambridge } \\
\text { Neuropsychological } \\
\text { Test Automated } \\
\text { Battery (CANTAB) }\end{array}$ & $\begin{array}{l}\text { The results showed that performance in both the fine and } \\
\text { gross motor skill task was significantly associated with better } \\
\text { performance in sustained attention }(p<0.001, p<0.001) \text {. } \\
\text { Higher exercise capacity was associated with fewer errors in } \\
\text { sustained attention }(-0.11 \pm 0.04, p=0.046) \text {. }\end{array}$ \\
\hline $\begin{array}{l}\text { Ciria et al. } \\
\text { (2017) [15] }\end{array}$ & $\begin{array}{l}\mathrm{N}=44 \text { (high-fit, } \mathrm{n}=22 \text { \& } \\
\text { low-fit, } \mathrm{n}=22) \\
\text { Age: }(18-23 \text { years old })\end{array}$ & $\begin{array}{l}\text { Incremental test } \\
\text { Frequency: once/session } \\
\text { Total session: } 1\end{array}$ & $\begin{array}{l}\text { Modified version } \\
\text { of Psychomotor } \\
\text { Vigilance Test (PVT) } \\
\text { \& Oddball test }\end{array}$ & $\begin{array}{l}\text { The mean RTs revealed a marginally significant main effect of } \\
\text { aerobic fitness, } \mathrm{F}(1,42)=3.663, \mathrm{p}=0.052, \mathrm{p}^{2}=0.08 \text {. } \\
\text { The high-fit group responded faster overall than low-fit group } \\
\text { ( } 323 \pm 24 \mathrm{~ms} \text { and } 346 \pm 46 \mathrm{~ms} \text {, respectively). }\end{array}$ \\
\hline $\begin{array}{l}\text { Chou et al. } \\
\text { (2017) [24] }\end{array}$ & $\begin{array}{l}\mathrm{N}=49 \text { (males and females) } \\
\text { Age: }(8-12 \text { years old) }\end{array}$ & $\begin{array}{l}\text { Yoga exercise (Heart rate) } \\
\text { Frequency: twice/week } \\
\text { Total sessions: } 16 \text { (8 weeks) }\end{array}$ & $\begin{array}{l}\text { Visual Pursuit Test } \\
\text { (VPT) }\end{array}$ & $\begin{array}{l}\text { The accuracy rate results showed that a significant interaction } \\
\text { of exercise group and control group by time was found, } \\
F(1,47)=4.26, p=0.045 \text {, partial } \eta^{2}=0.08 \text {. }\end{array}$ \\
\hline $\begin{array}{l}\text { Ballester et al. } \\
\text { (2017) [14] }\end{array}$ & $\begin{array}{l}\mathrm{N}=60 \text { (males, } \mathrm{n}=30 \& \\
\text { females, } \mathrm{n}=30) \\
\text { Age: } 11.0(0.2) \text { years old. }\end{array}$ & $\begin{array}{l}\text { Leger Multi-stage fitness } \\
\text { test } \\
\text { Frequency: once/session } \\
\text { Total sessions: } 1\end{array}$ & $\begin{array}{l}\text { Psychomotor } \\
\text { Vigilance Test (PVT) }\end{array}$ & $\begin{array}{l}\text { Reaction time: } \\
\text { Football players showed faster RTs than track and field athletes, } \\
\mathrm{t}(38)=3.01, \mathrm{p}=0.014, d=0.98 \text {, and non-athletes, } \mathrm{t}(38)=2.98 \text {, } \\
\mathrm{p}=0.014, d=0.97 \text {. } \\
\text { However, no significant differences were found between track } \\
\text { and field athletes and non-athletes ( } \mathrm{p}=0.311 \text { ) } \\
\text { Condition on reaction time (normal vs speed): } \\
\text { There was a significant main effect of condition on RTs } \\
\left(\mathrm{F}(2,54)=34.89, \mathrm{p}<0.001, \eta^{2} \text { partial }=0.39 \text { ). Participants were }\right. \\
\text { faster in the speed condition ( } 382 \mathrm{~ms} \text { ) than in the normal } \\
\text { condition }(432 \mathrm{~ms}) \text {. }\end{array}$ \\
\hline $\begin{array}{l}\text { Kato et al. } \\
\text { (2018) [19] }\end{array}$ & $\begin{array}{l}\mathrm{N}=23 \text { (males, } \mathrm{n}=13 \& \\
\text { females, } \mathrm{n}=10) \\
\text { Age: } 22.0(2.2) \text { years old }\end{array}$ & $\begin{array}{l}\text { Daily physical activity } \\
\text { (uniaxial accelerometer) } \\
\text { Duration: } 1 \text { week } \\
\text { Total sessions: - }\end{array}$ & $\begin{array}{l}\text { Continuous } \\
\text { Performance } \\
\text { Test-Identical Pairs } \\
\text { (CPT-IP) }\end{array}$ & $\begin{array}{l}\text { Exercise amount was not significantly correlated with \% correct } \\
\text { (mean } \pm S D=87.4 \pm 12.4, r=0.163, p=0.458) \text { and } d \text { prime } \\
\text { (mean } \pm S D=2.5 \pm 0.8, r=-0.036, p=0.869 \text { ) on CPT-IP. }\end{array}$ \\
\hline
\end{tabular}

$\mathrm{PA}=$ physical activity; $\mathrm{SS}=$ stretching session; Hit RT = hit reaction time; hit RT SE = hit reaction time standard error; Hit RT ISI change = hit reaction time interstimulus change; Hit SE ISI change $=$ hit standard error interstimulus change; SD = Standard deviation; MTP = methylphenidate drug; ERP = electrophysiological; $\%$ lapses = percentage of lapses; $\mathrm{RTs}=$ reaction time; \% correct = percentage of correct response; $d$ prime = signal detection index (discriminality from "hits" and "false alarm"). 


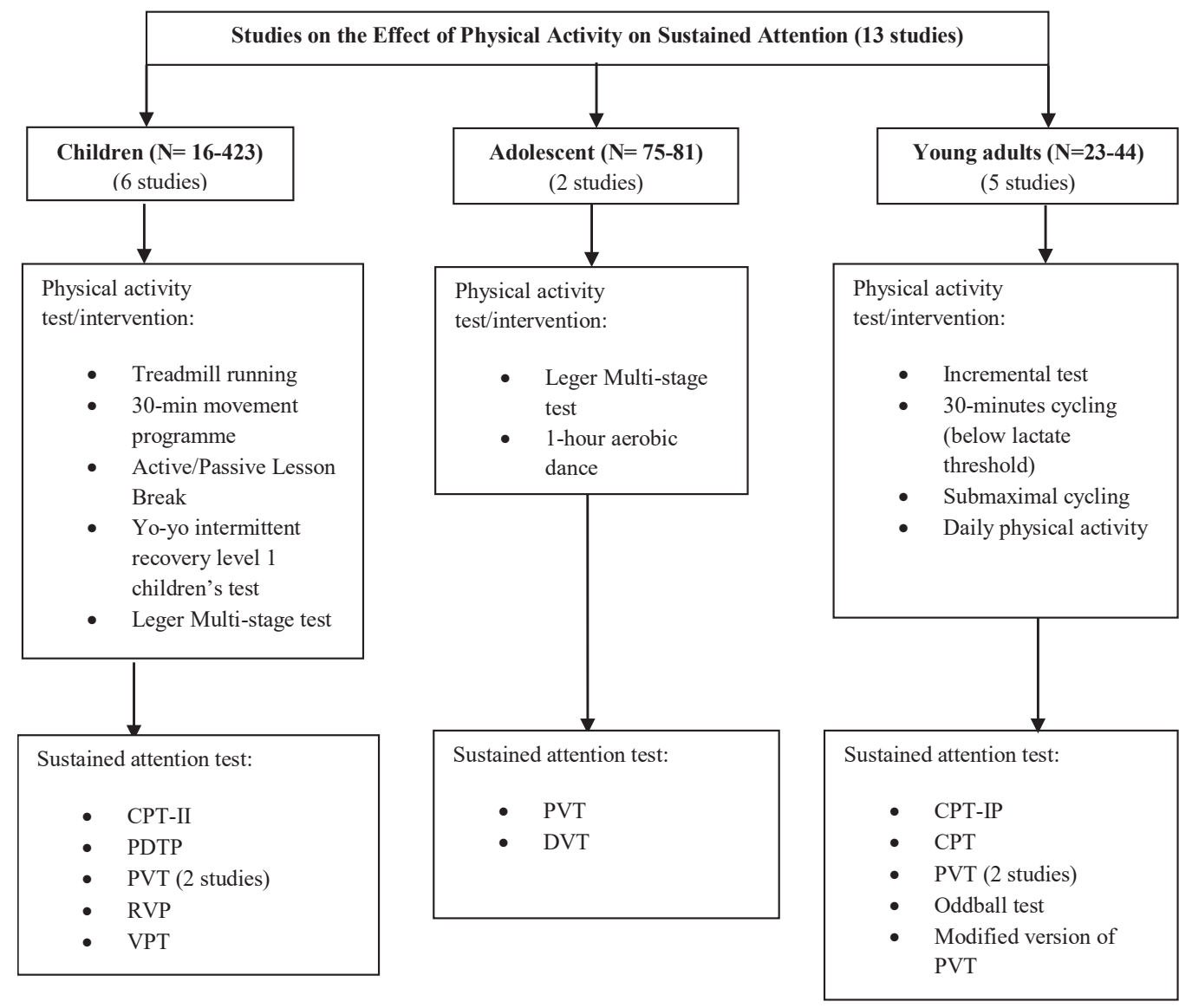

Figure 4. Summary of extracted study data from previous sustained attention studies.

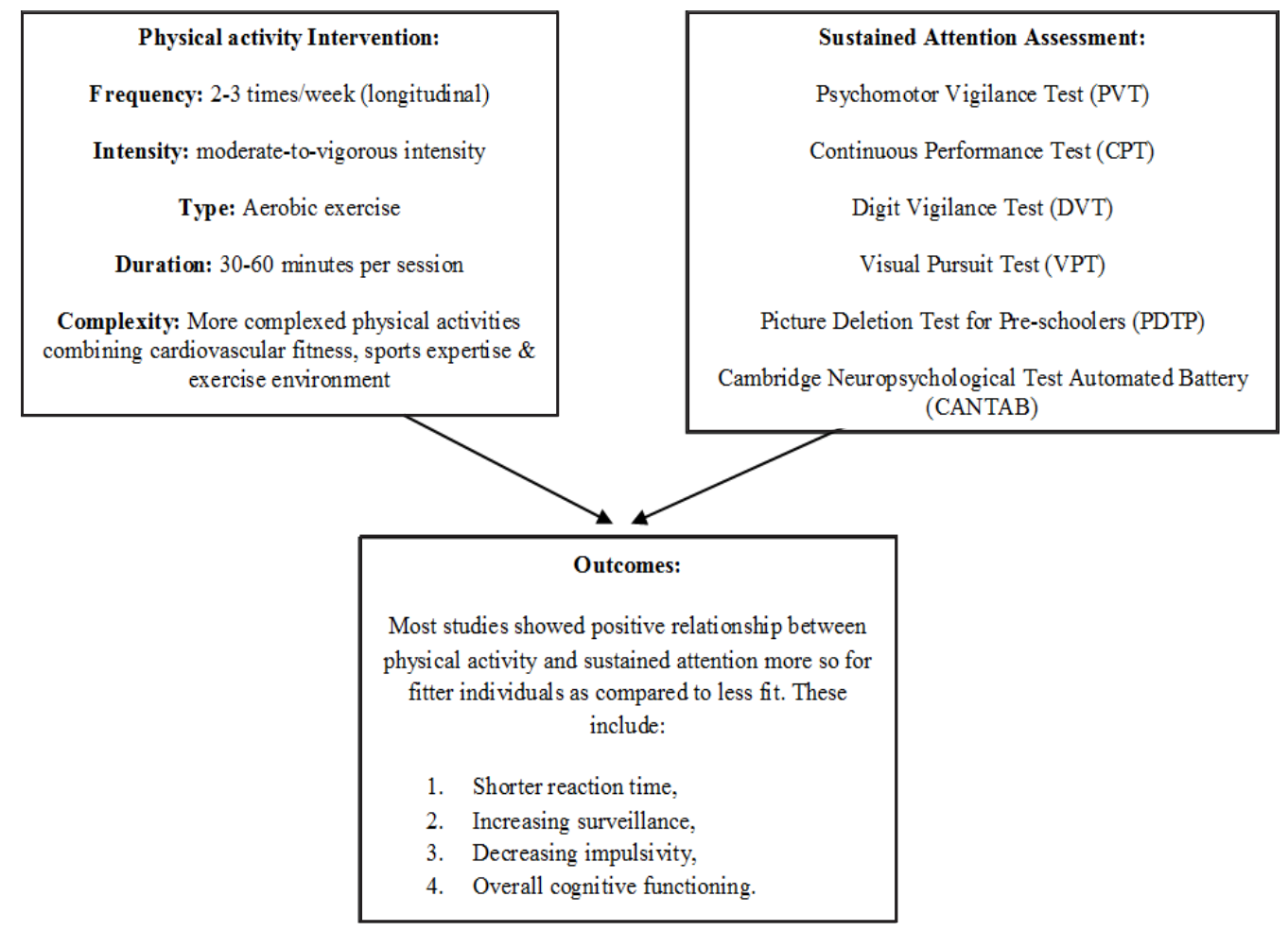

Figure 5. The positive outcomes from physical activity and sustained attention relationship. 


\section{DISCUSSION}

Based on the results obtained from the collected studies, physical activity has a positive impact on sustained attention. Individuals who are more fit showed better-sustained attention than individuals who are less fit. Researchers found that more fit individuals performed shorter reaction time, increased surveillance, less impulsive, and had a better overall cognitive functioning. Figure 5 demonstrates the summary of relationship between PA intervention and sustained attention. Possible causal relations between physical activity and sustained attention might be based on "cardiovascular fitness hypothesis" which propose that cardiovascular fitness is the physiological mediator that explains the cognitive benefits of physical activity. However, an extensive meta-regression [36] did not support this hypothesis and concluded that it is unrelated to cognitive performance. This metaregression suggested that other than cardiovascular fitness may affect the relationship between physical activity and cognitive performance. It is in line with the result found by Ballester et al. [15]. They found there were no significant correlations between the indices of vigilance performance (overall reaction time and lapses) and the main index of physical fitness (time-to-exhaustion); and no significant differences in performance in the vigilance assessment (PVT) between males and females, even though they were considerably different in cardiovascular fitness. The result becomes a question mark that cardiovascular fitness may not be the causal for cognitive improvement from physical activity. This may be an interesting topic to be studied to fill in the gap to understand the possible causal relations between cognitive functions and physical activity.

In addition, there are various of moderating factors that can affect the magnitude of the effects of physical activity and cognitive function relationship [37,38] such as mode or type of exercise (i.e. small effect in running vs. cycling), type of cognitive task (i.e. smaller effect on processing tasks vs. memory tasks), duration of exercise [39] and timing of the task following exercise. Furthermore, control rest condition can also affect the result [40]. Each of these factors should be taken into consideration by the researchers before delivering intervention to the subjects.

Researchers must know what kind of physical activity intervention to be delivered. First, we need to look at basic features of exercise; type or mode, intensity, frequency and duration of physical activity to give an effect on participants' sustained attention. Most of studies have applied moderate-to-high intensity aerobic physical or aerobic exercise that emphasises several features such as cardiovascular fitness, sports skills, and exercise environments $[41,42]$ while only one study carried out flexibility physical activity intervention [35], one study administered the daily PA by recording the number of steps and exercise amount per day from uniaxial accelerometer [16], and one study conducted active and passive lesson breaks [36]. It shows that PA intervention has progressively implemented into applicable settings such as during academic lesson breaks and daily life. This is crucial to see this physical execution not only from one setting, but it is also easier to be carried out in daily life at any time. It does not matter whether it is conducted in laboratory setting or field setting, both have shown a significant finding on sustained attention. There are also other types of PA such as 30-minutes movement programmed, aerobic dance, active and passive lesson breaks, daily physical activity and yoga. However, there was a lack of findings in implementing resistance exercise. Thus, future study may consider this type of exercise to examine its effect on sustained attention. In any case, aerobic capacity should be taken into consideration as it is the main key factor to determine the effectiveness of PA on sustained attention. Several findings have shown a significant outcome pertaining to the effect of MVPA on sustained attention $[5,15$, 23, 41-43]. Therefore, future research should consider administration of PA in accessible settings at the same time fulfil the PA guideline and recommendation from American College of Sports Medicine, American Heart Association and World Health Organization. Figure 4 shows the summary of extracted studies data related to the effect of PA on sustained attention.

Researches should consider specific sports groups and the account of PA for the potential influence of the perceptual-cognitive skills involved in sport training context to clarify the specific, rather than combined, effect for both cardiovascular fitness and the training on vigilance performance [31]. Therefore, as a suggestion for future studies, comparing larger groups of participants from different sport types and with different levels of sport participant and the participants' cardiovascular fitness are needed to clarify the specific, rather than combined effect of each variable on vigilance performance [14].

The physical activity implemented is between 30 to 60 minutes per session and is done at two to three times a week. These collective results are slightly different from the meta-analytical results [44] which recommended at least 20 minutes of exercise 
to achieve improvement in cognitive performance. However, in the sense of the intensity of exercise, both meta-analytical studies agree that exercise or physical activity with moderate-to-vigorous intensity gives a clearer impact on cognitive performance rather than low-intensity exercises.

In relation to the frequency and duration of exercise performed, every study has a variety of approach to ensure the target duration of exercise is achieved based on global guidelines. ACSM recommends participating in moderate-intensity endurance PA, for a minimum 30 minutes, five days per week; or participating in vigorous-intensity endurance PA for a minimum 20 minutes, three days per week to maintain a healthy lifestyle [3]. Aerobic exercises must be emphasised during PA sessions. Researchers should also pay attention to the type and number of subjects recruited. Some of the recruited subjects have other commitments that may affect their ability to keep on engaging in intervention and affect the duration of the study. Researchers must be aware that the involvement of health professionals as observers, if necessary, should be considered as well. For example, school children are committed to schooling hours while adults have work commitments. Meanwhile, the vulnerability of disabled people as compared to normal and healthy people requires parental consent before they can involve in any intervention, in addition to requiring the supervision of a doctor or a well-trained healthcare professional. Researchers should consider these aspects before deciding their study design.

It is speculated that due to methodological differences, especially the measurements used for sustained attention, have contributed to contradictory finding as it may not sensitive enough to the participants' changes in sustained attention [21]. Therefore, the selected measurement should be appropriate to the design and study objectives. The selection of the inappropriate measurements will affect the significance of the obtained results. Besides, researches may provide more challenging task to discriminate the participants' sustained attention as well as consider other factors, such as nutritional intake and duration of sleep to see its effect on the relationship between physical activity and sustained attention.

The most widely used sustained attention assessments are PVT. This assessment is available in various versions depending on the age of the subject and the objective of the study. The standard PVT only takes about 10 minutes. However, there is also a 60-minutes version used by Luque-Casado et al. [17] to assess the sustained attention of young adults.
Meanwhile, most PVT assessment versions were used on children and adolescents and took less than 9 minutes (minimum is 5 minutes). Among the possibilities of this assessment is often used by researchers are due to less complex and complicated variables to be measured and even the duration of the assessment takes only a short time. However, this assessment involves inhibition of responses to unpredictable stimuli, thus requiring less executive demand. Thus, PVT may risk in causing a lack of significant relationship between cardiovascular fitness and cognitive processing measure [44]. Therefore, researchers should know the appropriate assessment to be used based on the objectives and study design.

Next assessment that frequently used is the Continuous Performance Test. This assessment also has multiple versions depending on age and subjects' conditions. It takes at least 14 minutes to be done and the longest duration it can take is 20 minutes. Most of the assessment versions were used among young adults. However, there is another version that was used among attention-deficit hyperactivity disorder children (Continuous Performance Test - II), in a study conducted by Medina et al. [20] which has high number of variables. However, the variables in the standard Continuous Performance Test assessment is not as much as CPT-II. This assessment might not as easy as compared to PVT because it takes a longer duration to be done but these it has higher chance to demonstrate a significant relationship between physical and cognitive activities, regardless of other moderating factors [45].

Other suggestions for future study to examine the effect of the association between PA and sustained attention on academic performance which can produce a strong justification on the importance of sustaining attention. This observation may encourage teachers' interest to encourage their students to participate in daily physical activity by considering the implementation of short physical activity breaks as it becomes a concern about reduced attention due to the disruption to the classroom routine. To entice to education policymakers, research needs to cover a wider range of educational settings as well as include academic performance as a longer-term outcome. It is not only to create interest for future study but also to draw attention to the policymakers in promoting PA among grassroots.

It is also suggested that a study needs to be replicated in a wider range of schools and age ranges. As boys are more prone to inattention during extended academic study periods, the current findings may not generalise to girls [18]. More research is needed to determine 
the time course of changes over the longer term, and the extent to which ongoing time commitments and potential 'fatigue' among students and teachers impact the long-term implementation of the physical activity break $[39,46]$. In addition, both cognitive and academic tests suggest that it is important to focus on general motor skill development in school children. Future intervention studies focusing on motor skill training should investigate how improvements in fine and gross motor skills affect cognitive functions and academic performance in children and the possible underlying mechanisms should be investigated.

There was a limitation when studying the effects of PA on sustained attention is the consideration to vigilance decrement due to mental fatigue state which onsets after maintaining attention for a long duration [47]. It is noted that fitter individuals responded faster overall towards the psychomotor vigilance test than less fit individuals [15]. This may give rise to the hypothesis that athletes reach a state of mental fatigue much later as compared to normal individuals as they perform better in sustained related task in the early phases. Here, it is important to note that these previous studies used experimental tasks that typically last for only a few minutes (i.e., 10 minutes at the most), a duration that might be insufficient to elicit a significant deterioration in vigilance performance in young adults [48]. Thus, the duration of the task appears to be a key factor to pinpoint the link between aerobic fitness and sustained attention. Maintaining a high attentional state is effortful and it is taxed by time-on-task $[48,49]$. Alternatively, a habituation effect (i.e., a gradual familiarity effect and reduction of the cue-related heart response with repeated stimulus presentation), could also have been responsible for the disappearance of the observed cardiac deceleration in higher-fit individuals [50-52].

\section{CONCLUSION}

Sustained attention is one of the cognitive functions that are important for gathering information and instruction. Most of the papers in this review concluded that physical activity benefits the participants in sustaining their attention especially in aerobic exercises from moderate to high intensity. Future studies may consider studying the corresponding effect of gender, age, motivation and perceived arousal on this relationship to view other possible factors that can affect it indirectly; or even to try other types of exercise such as strength training and flexibility exercise to see whether it affects sustained attention.

\section{NOTES}

Funding

This research was supported by the Research University's Individual Grant (USM-RUI, 1001/PPSP/812149) from Universiti Sains Malaysia (USM). Hajar acknowledges the support from USM for awarding her the USM Graduate Assistant Scheme, and Rizal thanks the support from USM for awarding him the USM Fellowship Scheme.

\section{Conflicts of interest disclosure}

The authors declare no competing interests relevant to the content of this study.

\section{Authors' contributions.}

All the authors declare to have made substantial contributions to the conception, or design, or acquisition, or analysis, or interpretation of data; drafting the work or revising it critically for important intellectual content; to approve the version to be published.

Availability of data and responsibility for the results

All the authors declare to have had full access to the available data and they assume full responsibility for the integrity of these results.

\section{REFERENCES}

1. Caspersen CJ, Powell KE, Christenson GM. Physical activity, exercise, and physical fitness: definitions and distinctions for health-related research. Public Health Rep. 1985;100(2):126-31.

2. Ortega FB, Ruiz JR, Castillo MJ, Sjöström M. Physical fitness in childhood and adolescence: a powerful marker of health. Int J Obes. 2008;32(1):1-11. https://doi.org/10.1038/sj.ijo.0803774

3. Janssen I, Leblanc AG. Systematic review of the health benefits of physical activity and fitness in school-aged children and youth. Int J Behav Nutr Phys Act. 2010;7(1):40. https://doi.org/10.1186/1479-5868-7-40

4. Strong WB, Malina RM, Blimkie CJ, Daniels SR, Dishman RK, Gutin B, Hengenroeder AC, Must A, Nixon PA, Pivarnik JM, Rowland T, Trost S, Trudeau F. Evidence based physical activity for school-age youth. J Pediatr. 2005;146(6):732-7. https://doi.org/10.1016/j.jpeds.2005.01.055

5. Hillman CH, Erickson KI, Kramer AF. Be smart, exercise your heart: exercise effects on brain and cognition. Nat Rev Neurosci. 2008;9(1):58-65. https://doi.org/10.1038/nrn2298 
6. Best JR, Miller PH, Naglieri JA. Relations between executive function and academic achievement from ages 5 to 17 in a large, representative national sample. Learn Individ Differ. 2011;21(4):327-36. https://doi.org/10.1016/j. lindif.2011.01.007

7. Gillison FB, Skevington SM, Sato A, Standage M, Evangelidou S. The effects of exercise interventions on quality of life in clinical and healthy populations; a meta-analysis. Soc Sci Med. 2009;68(9):1700-10. https://doi.org/10.1016/j. socscimed.2009.02.028

8. Berger BG, Tobar DA. Physical activity and quality of life: key considerations. In: Tenenbaum G, Eklund RC, editors. Handbook of sport psychology. 3rd ed. Philadelphia: John Wiley \& Sons; 2007. p. 598-620. https://doi. org/10.1002/9781118270011.ch27

9. Kamijo K, Pontifex MB, O’Leary KC, Scudder MR, Wu CT, Castelli DM, Hillman CH. The effects of an afterschool physical activity program on working memory in preadolescent children. Dev Sci. 2011;14(5):1046-58. https://doi. org/10.1111/j.1467-7687.2011.01054.x

10. Mahar MT. Impact of short bouts of physical activity on attention-to-task in elementary school children. Prev Med. 2011;52 Suppl 1:S60-4. https://doi.org/10.1016/j.ypmed.2011.01.026

11. Cohen RA. Sustained attention. In: Kreutzer JS, DeLuca J, Caplan B, editors. Encyclopedia of clinical neuropsychology. New York: Springer; 2011. p. 2440-3.

12. Sarter M, Givens B, Bruno JP. The cognitive neuroscience of sustained attention: where top-down meets bottom-up. Brain Res Rev. 2001;35(2):146-60. https://doi.org/10.1016/s0165-0173(01)00044-3

13. Ballester R, Huertas F, Yuste FJ, Llorens F, Sanabria D. The relationship between regular sports participation and vigilance in male and female adolescents. PLoS One. 2015;10(4):e0123898. https://doi.org/10.1371/journal. pone. 0123898

14. Ballester R, Huertas F, Molina E, Sanabria D. Sport participation and vigilance in children: influence of different sport expertise. J Sport Health Sci. 2018;7(4):497-504. https://doi.org/10.1016/j.jshs.2017.05.008

15. Ciria LF, Perakakis P, Luque-Casado A, Morato C, Sanabria D. The relationship between sustained attention and aerobic fitness in a group of young adults. PeerJ. 2017;5:e3831. https://doi.org/10.7717/peerj.3831

16. Luque-Casado A, Perakakis P, Ciria LF, Sanabria D. Transient autonomic responses during sustained attention in high and low fit young adults. Sci Rep. 2016;6:27556. https://doi.org/10.1038/srep27556

17. Luque-Casado A, Perakakis P, Hillman CH, Kao SC, Llorens F, Guerra P, Sanabria D. Differences in sustained attention capacity as a function of aerobic fitness. Med Sci Sports Exerc. 2016;48(5):887-95. https://doi.org/10.1249/ mss.0000000000000857

18. Wilson AN, Olds T, Lushington K, Petkov J, Dollman J. The impact of 10-minute activity breaks outside the classroom on male students' on-task behaviour and sustained attention: a randomised crossover design. Acta Paediatr. 2016;105(4):e181-8. https://doi.org/10.1111/apa.13323

19. Kato K, Iwamoto K, Kawano N, Noda Y, Ozaki N, Noda A. Differential effects of physical activity and sleep duration on cognitive function in young adults. J Sport Health Sci. 2018;7(2):227-36. https://doi.org/10.1016/j.jshs.2017.01.005

20. Medina JA, Netto TL, Muszkat M, Medina AC, Botter D, Orbetelli R, Scaramuzza LF, Sinnes EG, Vilela M, Miranda MC. Exercise impact on sustained attention of ADHD children, methylphenidate effects. Atten Defic Hyperact Disord. 2010;2(1):49-58. https://doi.org/10.1007/s12402-009-0018-y

21. Leong IT, Moghadam S, Hashim HA. Aggregated effects of combining daily milk consumption and aerobic exercise on short-term memory and sustained attention among female students. Percept Mot Skills. 2015;120(1):57-66. https://doi.org/10.2466/22.06.pms.120v11x3

22. Palmer KK, Miller MW, Robinson LE. Acute exercise enhances preschoolers' ability to sustain attention. J Sport Exerc Psychol. 2013;35(4):433-7. https://doi.org/10.1123/jsep.35.4.433

23. Geertsen SS, Thomas R, Larsen MN, Dahn IM, Andersen JN, Krause-Jensen M, Korup V, Nielsen CM, Wienecke J, Ritz C, Krustrup P, Lundbye-Jensen J. Motor skills and exercise capacity are associated with objective measures of cognitive functions and academic performance in preadolescent children. PLoS One. 2016;11(8):e0161960. https://doi. org/10.1371/journal.pone.0161960

24. Chou C-C, Huang C-J. Effects of an 8-week yoga program on sustained attention and discrimination function in children with attention deficit hyperactivity disorder. PeerJ. 2017;5:e2883. https://doi.org/10.7717/peerj.2883 
25. Luque-Casado A, Zabala M, Morales E, Mateo-March M, Sanabria D. Cognitive performance and heart rate variability: the influence of fitness level. PLoS One. 2013;8(2):e56935. https://doi.org/10.1371/journal.pone.0056935

26. Mackworth NH. The breakdown of vigilance during prolonged visual search. Q J Exp Psychol. 1948;1(1):6-21. https://doi.org/10.1080/17470214808416738

27. Boksem MA, Meijman TF, Lorist MM. Effects of mental fatigue on attention: an ERP study. Brain Res Cogn Brain Res. 2005;25(1):107-16. https://doi.org/10.1016/j.cogbrainres.2005.04.011

28. Faber LG, Maurits NM, Lorist MM. Mental fatigue affects visual selective attention. PLoS One. 2012;7(10):e48073. https://doi.org/10.1371/journal.pone.0048073

29. Adan A. Cognitive performance and dehydration. J Am Coll Nutr. 2012;31(2):71-8. https://doi.org/10.1080/07315724. 2012.10720011

30. Sridharan D, Levitin DJ, Chafe CH, Berger J, Menon V. Neural dynamics of event segmentation in music: converging evidence for dissociable ventral and dorsal networks. Neuron. 2007;55(3):521-32. https://doi.org/10.1016/j. neuron.2007.07.003

31. Budde H, Voelcker-Rehage C, Pietrabyk-Kendziorra S, Ribeiro P, Tidow G. Acute coordinative exercise improves attentional performance in adolescents. Neurosci Lett. 2008;441(2):219-23. https://doi.org/10.1016/j.neulet.2008. 06.024

32. Roland AO. Effects of acute physical activity on sustained attention-the possible influence of natural environments [tese]. Trondheim: NTNU; 2017.

33. Caterino MC, Polak ED. Effects of two types of activity on the performance of second-, third-, and fourth-grade students on a test of concentration. Percept Mot Skills. 1999;89(1):245-8. https://doi.org/10.2466/pms.1999.89.1.245

34. Kalia R. A study of vigilance as related to gender and performance of aquatic players. Int J Acad Res Dev. 2018;3(1):976-79.

35. Etnier JL, Nowell PM, Landers DM, Sibley BA. A meta-regression to examine the relationship between aerobic fitness and cognitive performance. Brain Res Rev. 2006;52(1):119-30. https://doi.org/10.1016/j.brainresrev.2006.01.002

36. Cochran WG. Sampling techniques. 3rd ed. New York: John Wiley \& Sons; 1977.

37. Tomporowski PD. Effects of acute bouts of exercise on cognition. Acta Psychol (Amst). 2003;112(3):297-324. https://doi.org/10.1016/s0001-6918(02)00134-8

38. Brisswalter J, Collardeau M, René A. Effects of acute physical exercise characteristics on cognitive performance. Sports Med. 2002;32(9):555-66. https://doi.org/10.2165/00007256-200232090-00002

39. Chang YK, Chu CH, Wang CC, Wang YC, Song TF, Tsai CL, Etnier JL. Dose-response relation between exercise duration and cognition. Med Sci Sports Exerc. 2015;47(1):159-65. https://doi.org/10.1249/mss.0000000000000383

40. Lambourne K, Tomporowski P. The effect of exercise-induced arousal on cognitive task performance: a metaregression analysis. Brain Res. 2010;1341:12-24. https://doi.org/10.1016/j.brainres.2010.03.091 https://doi. org/10.1016/j.brainres.2010.03.091

41. Aberg MA, Pedersen NL, Torén K, Svartengren M, Bäckstrand B, Johnsson T, Cooper-Kuhn CM, Aberg ND, Nilsson M, Kuhn HG. Cardiovascular fitness is associated with cognition in young adulthood. Proc Natl Acad Sci U S A. 2009;106(49):20906-11. https://doi.org/10.1073/pnas.0905307106

42. Hillman CH, Castelli DM, Buck SM. Aerobic fitness and neurocognitive function in healthy preadolescent children. Med Sci Sports Exerc. 2005;37(11):1967-74. https://doi.org/10.1249/01.mss.0000176680.79702.ce

43. Hillman CH, Pontifex MB, Raine LB, Castelli DM, Hall EE, Kramer AF. The effect of acute treadmill walking on cognitive control and academic achievement in preadolescent children. Neuroscience. 2009;159(3):1044-54. https:// doi.org/10.1016/j.neuroscience.2009.01.057

44. Chang Y-K, Labban J, Gapin J, Etnier JL. The effects of acute exercise on cognitive performance: a meta-analysis. Brain Res. 2012;1453:87-101. https://doi.org/10.1016/j.brainres.2012.06.039

45. Majorek M, Tüchelmann T, Heusser P. Therapeutic Eurythmy-movement therapy for children with attention deficit hyperactivity disorder (ADHD): a pilot study. Complement Ther Nurs Midwifery. 2004;10(1):46-53. https://doi. org/10.1016/s1353-6117(03)00087-8 
46. Telles S, Singh N, Bhardwaj AK, Kumar A, Balkrishna A. Effect of yoga or physical exercise on physical, cognitive and emotional measures in children: a randomized controlled trial. Child Adolesc Psychiatry Ment Health. 2013;7(1):37. https://doi.org/10.1186/1753-2000-7-37

47. Warm JS, Parasuraman R, Matthews G. Vigilance requires hard mental work and is stressful. Hum Factors. 2008;50(3):433-41. https://doi.org/10.1518/001872008x312152

48. Grier RA, Warm JS, Dember WN, Matthews G, Galinsky TL, Parasuraman R. The vigilance decrement reflects limitations in effortful attention, not mindlessness. Hum Factors. 2003;45(3):349-59. https://doi.org/10.1518/ hfes.45.3.349.27253

49. Bradley MM. Natural selective attention: orienting and emotion. Psychophysiol. 2009;46(1):1-11. https://doi. org/10.1111/j.1469-8986.2008.00702.x

50. Sokolov EN. Higher nervous functions: the orienting reflex. Ann Rev Physiol. 1963;25:545-80. https://doi.org/10.1146/ annurev.ph.25.030163.002553

51. Thompson RF, Spencer WA. Habituation: a model phenomenon for the study of neuronal substrates of behavior. Psychol Rev. 1966;73(1):16-43. http://psycnet.apa.org/doi/10.1037/h0022681

52. Kumar N, Wheaton LA, Snow TK, Millard-Stafford M. Exercise and caffeine improve sustained attention following fatigue independent of fitness status. Fatigue. 2015;3(2):104-21. https://doi.org/10.1080/21641846.2015.1027553 